\title{
Does Crystallinity of Extracted Bone Mineral Increase over Storage Time?
}

\author{
William Querido $^{\mathrm{a}, \mathrm{b}}$, Andre L. Rossi ${ }^{\mathrm{a}}$, Andrea P. C. Campos ${ }^{\mathrm{c}}$, Alexandre M. Rossi ${ }^{\mathrm{d}}$, Marcos Farina ${ }^{\mathrm{a}, \mathrm{*}}$ \\ anstituto de Ciências Biomédicas, Universidade Federal do Rio de Janeiro - UFRJ, \\ CEP 21941-902, Rio de Janeiro, RJ, Brazil \\ ${ }^{\mathrm{b}}$ Instituto de Biofísica Carlos Chagas Filho, Universidade Federal do Rio de Janeiro - UFRJ, \\ CEP 21941-902, Rio de Janeiro, RJ, Brazil \\ ${ }^{\mathrm{C}}$ Divisão de Metrologia de Materiais, Instituto Nacional de Metrologia, Qualidade e Tecnologia, \\ CEP 25250-020, Duque de Caxias, RJ, Brazil \\ ${ }^{\mathrm{d}}$ Grupo de Biomateriais: Preparação, Caracterização, Modelagem Teórica e Aplicações Biomédicas, \\ Centro Brasileiro de Pesquisas Físicas, CEP 22290-180, Rio de Janeiro, RJ, Brazil \\ 'Instituto de Ciências Biomédicas, Centro de Ciências da Saúde, Universidade Federal do Rio de \\ Janeiro - UFRJ, Av. Carlos Chagas Filho, 373, bloco F, sala F2-027, Cidade Universitária,
} CEP 21941-902, Rio de Janeiro, RJ, Brazil

Received: December 13, 2012; Revised: January 30, 2013

\begin{abstract}
It was recently shown that the crystallinity of extracted bone mineral samples from the fin bones of zebrafish could increase over storage time. This would have implications in many studies in which the samples need to be stored until analysis. The aim of this study was to further evaluate if the crystallinity of extracted bone mineral increases over storage time. The extracted mineral was a biological bone-like apatite produced in osteoblast cell cultures. The overall characterization of the mineral was done by energy dispersive X-ray spectroscopy, Fourier transform infrared spectroscopy, and high-resolution transmission electron microscopy. In order to evaluate possible changes in crystallinity over storage time, the same sample was analyzed by X-ray diffraction immediately after mineral extraction and after 18 months of storage. In conclusion, no statistically relevant changes were observed over storage time, although the occurrence of a slight increase in crystallinity could be discussed in the stored mineral sample.
\end{abstract}

Keywords: bone mineral, crystallinity, material storage, $X$-ray diffraction, biomineralization

\section{Introduction}

The mineral phase found in bone can be generally described as a poorly crystalline, non-stoichiometric, $\mathrm{CO}_{3}{ }^{2-}$-containing hydroxyapatite ${ }^{1,2}$. However, the nanostructure of bone mineral may comprise not only poorly crystalline apatite, but also amorphous materials, especially amorphous calcium phosphate (ACP), which may act as a precursor phase of the apatite nanocrystals ${ }^{3-5}$. In this context, the overall crystallinity of bone mineral may reflect the relative proportions of crystalline and amorphous minerals. For instance, the increase in bone mineral crystallinity described during tissue maturation could be related to the transformation of ACP into apatite, leading to an increase in the relative proportion of crystalline to amorphous minerals ${ }^{3}$.

The mechanism by which poorly crystalline bone apatite is formed remains under dispute; however, recent studies have suggested that a sequence of non-classical crystallization stages, in which the collagen matrix play an essential role, may be involved in apatite nucleation and growth ${ }^{4,6,7}$. According to this model, ions from the surrounding matrix fluids would form pre-nucleation clusters and aggregate into round ACP nanoparticles, which would crystallize and act as precursors to the apatite nanocrystals. The formation of a disordered calcium phosphate precursor

*e-mail: mfarina@icb.ufrj.br may occur within intracellular vesicles of osteoblast cells, as shown in the developing mouse calvaria and long bones ${ }^{5}$. The formation of intracellular calcium phosphate was also recently described in osteoblast cell cultures, both within intracellular vesicles and mitochondrial granules ${ }^{8}$. ACP nanoparticles of 10-20 $\mathrm{nm}$ in diameter were described in the forming fin bones of zebrafish ${ }^{3,4}$, while crystalline round nanoparticles of about 2-3 $\mathrm{nm}$ in diameter were found in early stages of chicken bone crystal growth ${ }^{9}$ and in the newly formed bone around biomaterials ${ }^{10}$. Crystalline nanoparticles were also seen in murine femoral trabecular bone, being proposed to act as nuclei for the apatite nanocrystals ${ }^{11}$. The subsequent fusion of these crystalline nanoparticles would originate the plate-like bone apatite nanocrystals ${ }^{9}$. This fusion is in line with observations that bone apatite material is polycrystalline, which suggests the existence of multiple independent crystallization sites among the nanocrystals ${ }^{10-12}$.

While studying the forming fin bones of zebrafish, Mahamid et al. ${ }^{3}$ noticed that the extracted mineral phase showed a slight increase in crystallinity over storage time. The authors made this observation by depositing the mineral sample onto marked transmission electron microscopy grids and analyzing the same region immediately after 
extraction and again after storage of the grid for one week at room temperature. They examined the mineral by selected area electron diffraction (SAED) in a transmission electron microscope, and showed that the freshly extracted mineral from young, forming bones comprised particles with different degrees of crystallinity, including some that produced typical poorly crystalline diffraction patterns and other showing diffuse rings, indicating the presence of an amorphous material. Interestingly, by analyzing these latter particles after storage, they identified a slightly more crystalline sample, presenting faint spots corresponding to the (002) reflection of hydroxyapatite crystals.

In light of this observation by Mahamid et al. ${ }^{3}$, it is important to evaluate in more detail if the crystallinity of extracted bone mineral increases over storage time. Such change in the bone mineral phase could have relevant implications for many studies, since the mineral samples often cannot be fully characterized immediately after extraction and/or need to be stored for further analyses.

\section{Material and Methods}

The biological bone-like mineral used in this study was produced in cell culture systems using F-OST osteoblast cells, previously isolated from the endosteal region of murine femurs ${ }^{13}$. The F-OST osteoblast cell cultures were recently shown by a variety of methods to produce a mineralized matrix with many bone-like ultra-structural and mineral phase features, being a suitable model for bone mineralization studies ${ }^{14}$. The cells were cultured for 28 days in Dulbecco's Modified Eagle's Medium (DMEM) containing $10 \%$ fetal bovine serum (FBS) and antibiotics (100 U/mL penicillin and $100 \mu \mathrm{g} / \mathrm{mL}$ streptomycin) at $37^{\circ} \mathrm{C}$ in a humidified $5 \% \mathrm{CO}_{2}$ incubator. In order to accelerate matrix formation and mineralization, the medium was supplemented with $50 \mu \mathrm{g} / \mathrm{mL}$ ascorbic acid and $10 \mathrm{mM}$ $\beta$-glycerophosphate.

The mineral produced in osteoblast cell cultures was extracted and analyzed as previously described ${ }^{14}$. Briefly, the mineral was extracted by removal of the organic matrix based on methods found to preserve main features of the bone mineral component ${ }^{3,15}$. The organic matrix was degraded by exposing the cell cultures to a $5 \% \mathrm{NaClO}$ solution for about 10 minutes in continuous motion in a vortex mixer. The suspension was centrifuged, the supernatant was discarded, and the mineral pellets were washed three times with Milli-Q water and twice with $100 \%$ ethanol, and dried overnight at $40{ }^{\circ} \mathrm{C}$. It is important to mention that this extraction and drying procedures could have led to slight changes in the original mineral component. The mineral extracted from five cell cultures was pooled into one powdered sample before analysis. The overall characterization of the mineral samples was done by the combination of methods described below, aiming to confirm the bone-like nature of the mineral phase produced in osteoblast cell cultures.

For energy dispersive X-ray spectroscopy (EDS), a small amount of the mineral was mixed with $100 \%$ ethanol, deposited on formvar-coated copper grids, and analyzed in an FEI Titan 80-300 transmission electron microscope equipped with an EDAX EDS System, at $300 \mathrm{kV}$. For Fourier transform infrared spectroscopy (FTIR), about $1 \mathrm{mg}$ of the extracted mineral was mixed with $100 \mathrm{mg}$ of $\mathrm{KBr}$, pressed into a thin disc, and analyzed in a Shimadzu IRPrestige-21 spectrometer. For high-resolution transmission electron microscopy (HRTEM), a small amount of the mineral was mixed with $100 \%$ ethanol, sonicated, deposited on Ted Pella, Inc ultrathin carbon film supported by a lacey carbon film on 400 mesh copper grids, and observed in a JEM 3010 URP microscope at $300 \mathrm{kV}$, operating at the Brazilian Synchrotron Light Laboratory, Campinas, Brazil.

To compare the crystallinity of the mineral over storage time, the same exact sample was analyzed by X-ray powder diffraction (XRD) immediately after extraction and over 18 months of storage. The mineral sample was stored in a dry, powdered state at room temperature under ambient conditions in a closed Eppendorf microtube. XRD was chosen for this analysis because it directly accesses the long-range structural order of a relatively large amount of material, allowing estimation of the overall degree of crystallinity of the mineral samples.

Briefly, about $60 \mathrm{mg}$ of extracted mineral was analyzed in a PANalytical X'Pert PRO diffractometer using $\mathrm{CuK}_{\alpha}$ radiation $(\lambda=1.5418 \AA$ ). The patterns were acquired with a step size of $0.02^{\circ} 2 \theta$ and counting time of $5 \mathrm{sec} / \mathrm{step}$. The diffraction peaks were indexed based on comparison with standard Powder Diffraction Files (PDF) from the PDF-2 database of the International Centre for Diffraction Data (ICDD), particularly, with that of hydroxyapatite (ICDD PDF 9-432). Crystallinity analysis was done based on the full width at half maximum (FWHM) of the (002) reflection, obtained by fitting the peak with a Lorentz function using the OriginPro software version 8 . The degree of crystallinity $X c$ was estimated using the FWHM $\beta_{1 / 2}$ in degrees, employing the following Equation ${ }^{16}$ :

$$
\sqrt[3]{X c}=\frac{0.24}{\beta_{1 / 2}}
$$

The range of each measurement was estimated using the step size value as the uncertainty of the calculated FHWM, similar to that described previously ${ }^{17}$. Differences were considered relevant when the value range did not overlap.

\section{Results}

The general characteristics of the extracted mineral phase found in this study were in line with results previously described in the herein employed in vitro bone mineralization mode ${ }^{14}$. Briefly, EDS analysis showed $\mathrm{Ca}$ and $\mathrm{P}$ as main components of the mineral, with small amounts of $\mathrm{Mg}$ and $\mathrm{Na}$ also seen (Figure 1a). The mineral was identified by FTIR as a poorly crystalline, $\mathrm{CO}_{3}{ }^{2-}$-containing apatite, presenting bands typically described in hydroxyapatite phases $^{18}$ (Figure 1b). The $\mathrm{v}_{3} \mathrm{PO}_{4}{ }^{3-}\left(1200-900 \mathrm{~cm}^{-1}\right)$ appeared as a broad band with a discrete shoulder; the $v_{1} \mathrm{PO}_{4}{ }^{3-}\left(980-940 \mathrm{~cm}^{-1}\right)$ band was mostly overlapped with the $\mathrm{v}_{3} \mathrm{PO}_{4}^{3-}$; and the $\mathrm{v}_{4} \mathrm{PO}_{4}^{3-}\left(650-500 \mathrm{~cm}^{-1}\right)$ was partially resolved into two broad peaks. These shapes of the $\mathrm{PO}_{4}{ }^{3-}$ bands indicate the low crystallinity of the minerals. The presence of $\mathrm{CO}_{3}^{2-}$ was attributed to the clear bands of the $v_{3} \mathrm{CO}_{3}{ }^{2-}\left(1600-1350 \mathrm{~cm}^{-1}\right)$ and $\mathrm{v}_{2} \mathrm{CO}_{3}{ }^{2-}\left(890-850 \mathrm{~cm}^{-1}\right)$. Closer analysis of the $\mathrm{CO}_{3}{ }^{2-}$ bands indicates the presence 
of $\mathrm{CO}_{3}{ }^{2-}$ replacing both $\mathrm{PO}_{4}{ }^{3-}$ (type- $\mathrm{B}$ substitution) and $\mathrm{OH}^{-}$groups (type-A substitution), with a higher content seen in $\mathrm{PO}_{4}^{3-}$-sites, as previously describe in more detail ${ }^{14}$. Broad bands of the $v_{1} \mathrm{H}_{2} \mathrm{O}$ and $v_{3} \mathrm{H}_{2} \mathrm{O}\left(3700-2500 \mathrm{~cm}^{-1}\right)$ and $v_{2} \mathrm{H}_{2} \mathrm{O}\left(1700-1600 \mathrm{~cm}^{-1}\right)$ were also seen. Peaks of the $v_{1} \mathrm{OH}^{-}\left(3572 \mathrm{~cm}^{-1}\right)$ and $v_{\mathrm{L}} \mathrm{OH}^{-}\left(630 \mathrm{~cm}^{-1}\right)$ were not observed in the FTIR spectra, similar to results on bone apatite ${ }^{19}$. Observation of the mineral by HRTEM confirmed the poor long-range structural order of the mineral, which comprised nanocrystalline particles in conjunction with amorphous materials (Figure 1c, arrow and asterisk, respectively).

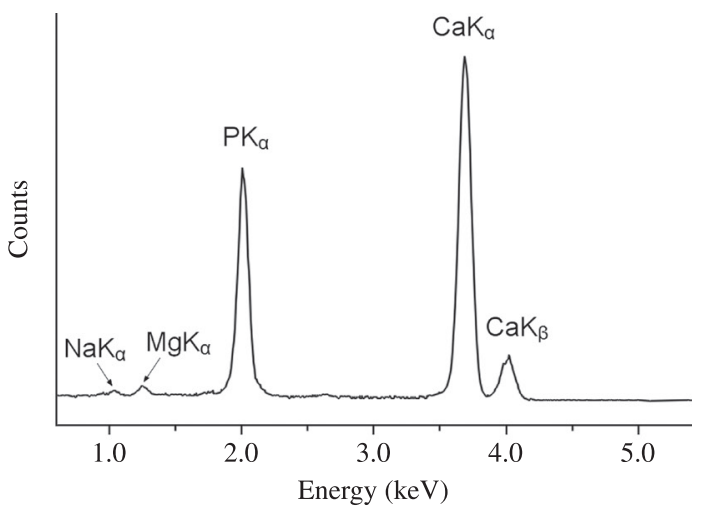

(a)

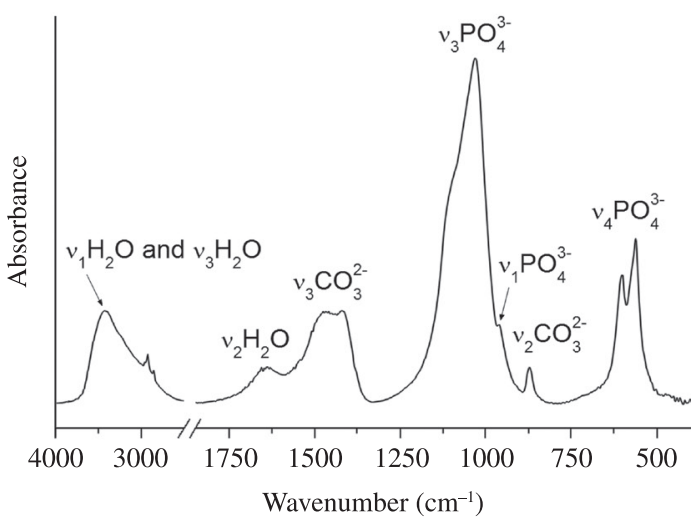

(b)

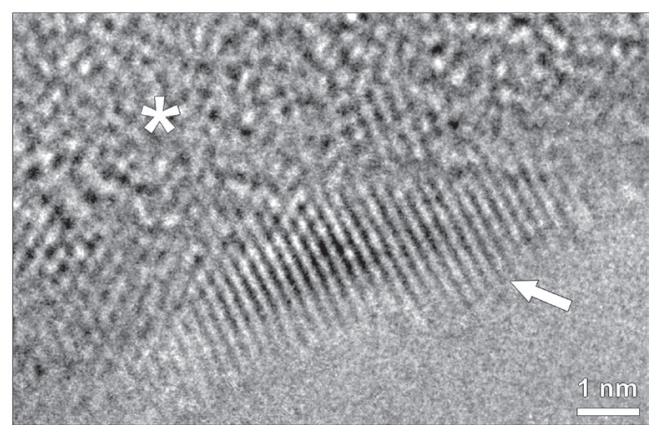

(c)

Figure 1. General characterization of the mineral sample. (a) EDS spectrum. Ca and $\mathrm{P}$ were main components of the mineral. (b) FTIR spectrum. The mineral was identified as a poorly crystalline, $\mathrm{CO}_{3}{ }^{2-}$-containing apatite. (c) HRTEM micrograph. The mineral comprised both nanocrystalline particles (arrow) and amorphous materials (asterisk).
In order to evaluate if the crystallinity of the extracted bone-like mineral could change over storage time, the same sample was analyzed by XRD immediately after mineral extraction and after 18 months of storage. In both cases, the mineral was identified as a poorly crystalline apatite, yielding XRD patterns very similar to those described for bone mineral $^{1}$ (Figure $2 \mathrm{a}$ ). Due to the low crystallinity of the samples, the patterns comprised broad, overlapping peaks, indexed, according to the ICDD PDF 9-432, as reflections from hydroxyapatite lattice planes. Among these, only the (002) reflection was an isolated, well-defined peak, and was used to compare the long range structural order of the samples. For this purpose, the (002) reflection was investigated by curve-fitting analysis (Figure $2 b$ ), and the FWHM values obtained were employed to estimate mineral crystallinity. No statistically relevant changes were noticed in the degree of crystallinity of the mineral after 18 months

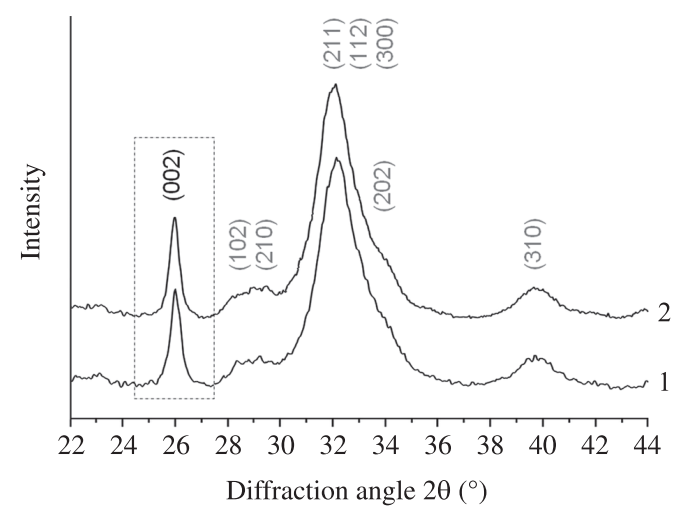

(a)

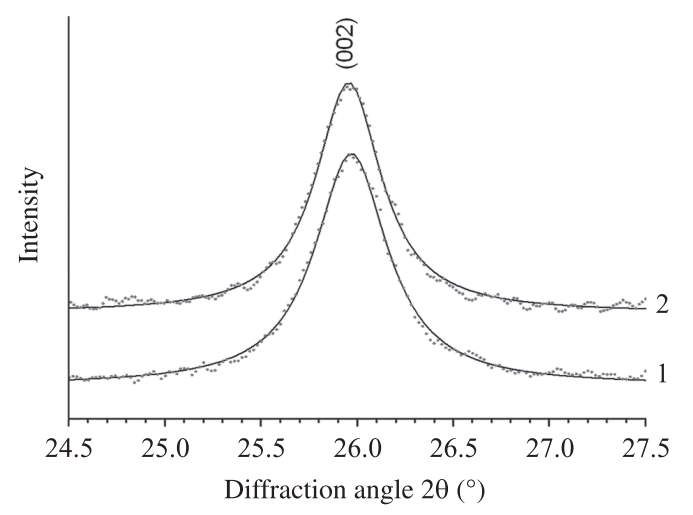

(b)

\section{1 - Initial sample $\quad 2$ - Sample after 18 months}

Figure 2. XRD analyses of the mineral sample over storage time. (a) XRD patterns of the initial sample (1) and of the same sample after 18 months of storage (2). Both patterns showed similar features, comprising broad, overlapping peaks indexed as reflections of hydroxyapatite planes (ICDD PDF 9-432). The (002) reflection was the only isolated, well-defined peak, being used to further analysis. (b) Curve-fitting analysis of the (002) reflection of the initial sample (1) and the same sample after 18 months of storage (2). Dotted line: experimental peak; Full line: fitted peak. The FWMH values obtained by this analysis were used to estimate the degree of crystallinity of the samples, as presented in Table 1. 
Table 1. Crystallinity analysis of the mineral sample over storage time. The FWHM of the (002) reflection was obtained by curvefitting analysis of the XRD peaks (see Figure $2 b$ ). No statistically relevant changes were observed in the mineral sample after 18 months of storage. However, the occurrence of a slight increase in the crystallinity of the stored sample may be discussed. FWHM are presented as calculated values \pm step size; crystallinity degrees are estimated as calculated values \pm range.

\begin{tabular}{ccc}
\hline & $\begin{array}{c}\text { FWHM of the } \\
(\mathbf{0 0 2}) \text { reflection }\left(^{(}\right)\end{array}$ & $\begin{array}{c}\text { Crystallinity degree } \\
\text { (arbitrary units) }\end{array}$ \\
\hline $\begin{array}{c}\text { Initial sample } \\
\text { Sample after 18 } \\
\text { months }\end{array}$ & $0.44 \pm 0.02$ & $0.81 \pm 0.02$ \\
\hline
\end{tabular}

of storage (Table 1). However, a possible slight decrease in the FWHM of the (002) reflection, and hence an increase in the crystallinity of the mineral over time may be discussed.

\section{Discussion}

The mineral phase analyzed in this study was quite similar to that described in native bone tissue, defined as a poorly crystalline, non-stoichiometric, $\mathrm{CO}_{3}{ }^{2-}$-containing hydroxyapatite ${ }^{1,2}$. Similar results were presented in more detail in a previous characterization of the mineral phase produced in the in vitro biomineralization model used in this study ${ }^{14}$. This model was herein found suitable for its purpose, allowing evaluation of the changes in the long-range structural order of a bone-like apatite over storage time. Although no relevant changes were noticed in this study, it was possible to recognize a slight, statistically insignificant increase in the degree of crystallinity of the mineral after 18 months of storage. An increase in the crystallinity of the mineral over time would be in line with that previously shown in the extracted mineral from the forming fin bones of zebrafish ${ }^{3}$.

It may be proposed that a possible increase in the crystallinity of extracted bone mineral over storage time could be associated with two main processes. The first, suggested by Mahamid et al. ${ }^{3}$, could involve the crystallization of a transient ACP precursor phase present in the mineral material. The conversion of this metastable amorphous phase into poorly crystalline bone apatite would increase the proportion of crystalline mineral relative to amorphous material, and hence increase the overall crystallinity of the mineral sample. The second process could be related to a direct increase in the degree of crystallinity of the initially poorly crystalline phase. These processes could occur individually or simultaneously, having somewhat different effects on the XRD patterns of the mineral. In the present study, since the XRD analyses were based on the (002) reflection of the crystalline apatite phase, the latter scenario may be more closely associated with the slight, statistically insignificant increase discussed to occur in the crystallinity of the mineral sample over storage time. In order to evaluate the proportion of crystalline to amorphous materials, the XRD patterns should be refined (e.g., by Rietveld refinement), which was not possible in this study due to the low resolution of the patterns.

It is important to consider that not all bones are the same, presenting, for instance, differences in structure and composition among distinct bone types, maturation stages, and animal species and age. Thus, extracted mineral samples from different bones or even regions within the same bone could have distinct behaviors over storage time. For instance, in the forming fin bones of zebrafish, the immature portions were found to present relatively higher amounts of ACP than the mature segments, which were more crystalline ${ }^{3}$. In this context, it is possible that the extracted mineral from the immature portions of the fin bones could present a higher relative increase in crystallinity over storage time than that of the mineral extracted from the mature segments. This result could be related to the different amounts of ACP found in the tissues, such that the more ACP is present in bone, the more noticeable would be its subsequent crystallization in the overall crystallinity of the extracted mineral sample.

In order to compare the results shown herein with those described previously by Mahamid et al. ${ }^{3}$, it is important to point out some relevant differences between these two studies, especially regarding the use of different model systems and analytical strategies. As discussed above, minerals from different bones may have distinct behaviors over storage time. In this context, it is conceivable that the in vitro mineralization model used in the present study could produce a mineral content slightly different than that found in the young forming bones used by Mahamid et al. ${ }^{3}$, resulting thus in differences in the changes observed in the degree of crystallinity of the minerals over storage time. Moreover, care should be taken when comparing results obtained herein by XRD with those obtained by Mahamid et al. ${ }^{3}$ by SAED. While XRD analysis results in the overall characterization of a relatively large amount of powdered sample, SAED results in the punctual analysis of mineral particles at the nanometer scale. For this reason, it is possible that, due to the small amount of amorphous material present in the overall mineral samples, bulk XRD analysis might not fully reproduce observations made locally by SAED in particles from young forming bones where relatively larger amounts of a metastable amorphous phase crystallizes into apatite over time, as those described by Mahamid et al. ${ }^{3}$.

In conclusion, no statistically relevant changes were observed in the crystallinity of the extracted bone-like mineral over 18 months of storage, although a slight increase could be discussed to occur in the XRD-evaluated crystallinity of the sample over time. In this context, it appears that the mineral extracted from the cell cultures employed herein could be relatively safely stored in a dry, powdered state for future analyses, which can be advantageous in numerous studies in the bone mineralization field. However, it is important to explicit that this conclusion might not be generalized, and, as discussed above, different types of bone mineral (e.g., from young forming bones vs. from mature bones) analyzed at distinct scales (e.g., overall measurements by XRD vs. local measurements at the nanometer scale by SAED) could yield slightly different results. Additionally, it should be mentioned that care should be taken when comparing stored samples with those recently extracted, and that it would be interesting to investigate in more detail, for instance, by Synchrotron XRD, the possible change in the mineral crystallinity over storage time in mineral samples extracted from different types of bones and 
in cases in which whole bones were stored and the mineral were extracted at a later date.

\section{Acknowledgements}

The authors gratefully acknowledge C.L.R. Fragoso and V.C.A. Moraes (Brazilian Center for Physics Research) for

\section{References}

1. Rey C, Combes C, Drouet C and Glimcher MJ. Bone mineral: update on chemical composition and structure. Osteoporosis International. 2009; 20(6):1013-21. PMid:19340505 PMCid:2760485. http://dx.doi.org/10.1007/s00198-0090860-y

2. Dorozhkin SV. Calcium orthophosphates in nature, biology and medicine. Materials. 2009; 2:399-498. http://dx.doi. org/10.3390/ma2020399

3. Mahamid J, Sharir A, Addadi L and Weiner S. Amorphous calcium phosphate is a major component of the forming fin bones of zebrafish: Indications for an amorphous precursor phase. Proceedings of the National Academy of Sciences USA. 2008; 105(35):12748-53. PMid:18753619 PMCid:2529085. http://dx.doi.org/10.1073/pnas.0803354105

4. Mahamid J, Aichmayer B, Shimoni E, Ziblat R, Li C, Siegel $\mathrm{S}$ et al. Mapping amorphous calcium phosphate transformation into crystalline mineral from the cell to the bone in zebrafish fin rays. Proceedings of the National Academy of Sciences USA. 2010; 107(14):6316-21. PMid:20308589 PMCid:2851957. http://dx.doi.org/10.1073/pnas.0914218107

5. Mahamid J, Sharir A, Gur D, Zelzer E, Addadi L and Weiner $\mathrm{S}$. Bone mineralization proceeds through intracellular calcium phosphate loaded vesicles: a cryo-electron microscopy study. Journal of Structural Biology. 2011; 174(3):527-35. PMid:21440636. http://dx.doi.org/10.1016/j.jsb.2011.03.014

6. Nudelman F, Pieterse K, George A, Bomans PH, Friedrich H, Brylka LJ et al. The role of collagen in bone apatite formation in the presence of hydroxyapatite nucleation inhibitors. Nature Materials. 2010; 9(12):1004-9. PMid:20972429 PMCid:3084378. http://dx.doi.org/10.1038/nmat2875

7. Colfen H. Biomineralization: A crystal-clear view. Nature Materials. 2010; 9(12):960-1. PMid:21102512. http://dx.doi. org/10.1038/nmat2911

8. Boonrungsiman S, Gentleman E, Carzaniga R, Evans ND, McComb DW, Porter AE et al. The role of intracellular calcium phosphate in osteoblast-mediated bone apatite formation. Proceedings of the National Academy of Sciences USA. 2012; 109(35):14170-5 PMid:22879397 PMCid:3435222. http://dx.doi.org/10.1073/pnas.1208916109

9. Cuisinier FJG, Steuer P, Brisson A and Voegel JC. High resolution electron microscopy study of crystal growth mechanisms in chicken bone composites. Journal of Crystal Growth. 1995; 156:443-53. http://dx.doi.org/10.1016/00220248(95)00237-5 assistance in the FTIR and XRD analyses, respectively, and LME/LNNano/CNPEM (Campinas, Brazil) for HRTEM performed on the JEM-3010 URP. We also thank INMETRO (Duque de Caxias, Brazil). This study was supported by CNPq, CAPES, FAPERJ, and FINEP (Brazilian agencies) and by the CAPES/COFECUB program n ${ }^{\circ} 628 / 09$ (Brazil/ France cooperation).

10. Rossi AL, Barreto IC, Maciel WQ, Rosa FP, Rocha-Leao MH, Werckmann J et al. Ultrastructure of regenerated bone mineral surrounding hydroxyapatite-alginate composite and sintered hydroxyapatite. Bone. 2012; 50(1):301-10. PMid:22057083. http://dx.doi.org/10.1016/j.bone.2011.10.022

11. Hong SI, Hong SK and Kohn DH. Nanostructural analysis of trabecular bone. Journal of Materials Science Materials in Medicine. 2009; 20:1419-26. PMid:19266266. http://dx.doi. org/10.1007/s10856-009-3708-2

12. Landis WJ, Song MJ, Leith A, McEwen L and McEwen BF. Mineral and organic matrix interaction in normally calcifying tendon visualized in three dimensions by high-voltage electron microscopic tomography and graphic image reconstruction. Journal of Structural Biology. 1993; 110(1):39-54. PMid:8494671. http://dx.doi.org/10.1006/jsbi.1993.1003

13. Balduino A, Hurtado SP, Frazão P, Takiya CM, Alves LM, Nasciutti LE et al. Bone marrow subendosteal microenvironment harbours functionally distinct haemosupportive stromal cell populations. Cell and Tissue Research. 2005; 319(2):255-266. PMid:15578225. http://dx.doi.org/10.1007/s00441-004-1006-3

14. Querido W, Abraçado LG, Rossi AL, Campos APC, Rossi AM, San Gil RAS et al. Ultrastructural and mineral phase characterization of the bone-like matrix assembled in F-OST osteoblast cultures. Calcified Tissue International. 2011; 89(5):358-71. PMid:21901516. http:// dx.doi.org/10.1007/s00223-011-9526-9

15. Weiner S and Price PA. Disaggregation of bone into crystals. Calcified Tissue International. 1986; 39(6):365-75. PMid:3026591. http://dx.doi.org/10.1007/BF02555173

16. Li ZY, Lam WM, Yang C, Xu B, Ni GX, Abbah SA et al. Chemical composition, crystal size and lattice structural changes after incorporation of strontium into biomimetic apatite. Biomaterials. 2007; 28(7):1452-60. PMid:17140655. http://dx.doi.org/10.1016/j.biomaterials.2006.11.001

17. Tong J, Clark D, Hoban M and O'Hayre R. Cost-effective solidstate reactive sintering method for high conductivity proton conducting yttrium-doped barium zirconium ceramics. Solid State Ionics. 2010; 181:496-503. http://dx.doi.org/10.1016/j. ssi.2010.02.008

18. Koutsopoulos S. Synthesis and characterization of hydroxyapatite crystals: a review study on the analytical methods. Journal of Biomedical Materials Research. 2002; 62(4):600-2. PMid:12221709. http://dx.doi.org/10.1002/jbm.10280

19. Rey C, Miquel JL, Facchini L, Legrand AP and Glimcher MJ. Hydroxyl groups in bone mineral. Bone. 1995; 16(5):583-586. http://dx.doi.org/10.1016/8756-3282(95)00101-I 